\title{
EVALUATION OF REAL DATA CALL SET UP SUCCESS RATE IN E-UTRAN
}

\author{
Martin KOLLÁR \\ CMT OP NW TAR, Siemens Program and System Engineering, Ltd., \\ Trieda SNP 37, 04100 Košice, Slovak Republic, tel.: +421 557953 243, e-mail: martin.kollar@siemens.com
}

\begin{abstract}
The Call Set up Success Rate (CSSR) is one of the most important Key Performance Indicators (KPIs) used by all mobile operators. The CSSR in general is a term in telecommunications denoting the fraction of the attempts to make a call which result in a connection to the dialled number. However there is no standard measurement possible for this parameter. Therefore the different operators can measure it differently.

In this paper, a definition of a real data CSSR, possibility of its implementation using the current technologies in LTE (Long Term Evolution) is provided and the obtained formula proposal is discussed as well. The real in this case means that data CSSR is calculated as ratio of the assigned Evolved Packet System (EPS) bearers to the random access preamble requests related to data calls with excluded ghosts, signaling radio bearer drops during call set up caused by Evolved Packet Core (EPC) network problems. The data means that the LTE is only packet switched based network, i.e. there are not any circuit switched services as it is typical for example for GSM. The proposed modeling can help mobile operators to measure the data CSSR properly.
\end{abstract}

Keywords: LTE, Call Set up Success Rate, EPS Bearer, RA Preamble

\section{INTRODUCTION}

The recent increase of mobile data usage and emergence of new applications such as MMOG (Multimedia Online Gaming), mobile TV, Web 2.0, streaming contents have motivated the 3rd Generation Partnership Project (3GPP) to work on the Long Term Evolution (LTE).

LTE, whose radio access is called Evolved UMTS Terrestrial Radio Access Network (E-UTRAN), is expected to substantially improve end-user throughputs, sector capacity and reduce user plane latency, bringing significantly improved user experience with full mobility. LTE has been set aggressive performance requirements that rely on physical layer technologies, such as, Orthogonal Frequency Division Multiplexing (OFDM) and Multiple-Input Multiple-Output (MIMO) systems, smart antennas to achieve these targets.

With the emergence of Internet Protocol (IP) as the protocol of choice for carrying all types of traffic, LTE is scheduled to provide support for IP-based traffic with endto-end Quality of Service (QoS). Voice traffic will be supported mainly as Voice over IP (VoIP) enabling better integration with other multimedia services [1].

\section{STRUCTURE OF LTE}

As mentioned in the chapter 1, LTE has been designed to support only packet switched services, in contrast to the circuit switched models of previous cellular systems. While the term LTE encompasses the evolution of the radio access through E-UTRAN, it is accompanied by an evolution of the non-radio aspects under the term System Architecture Evolution (SAE) which includes the Evolved Packet Core (EPC) network. Together E-UTRAN and SAE comprise the Evolved Packet System (EPS). The EPS architecture consists of the following functional elements as shown in Fig. 1 which are briefly described in this section (a very detailed description of LTE network elements can be found in [1]). In Fig. 1, SGi represents a reference point between the PDN Gateway and the operator's IP services like IP Multimedia Subsystem (IMS), Packet Switch Streaming (PSS) etc.

\section{Evolved Radio Access Network (E-UTRAN)}

E-UTRAN represents the access network of LTE which is a network of eNodeBs. For normal user traffic there is no centralized controller in E-UTRAN, i.e. the EUTRAN architecture is considered to be flat. The Evolved

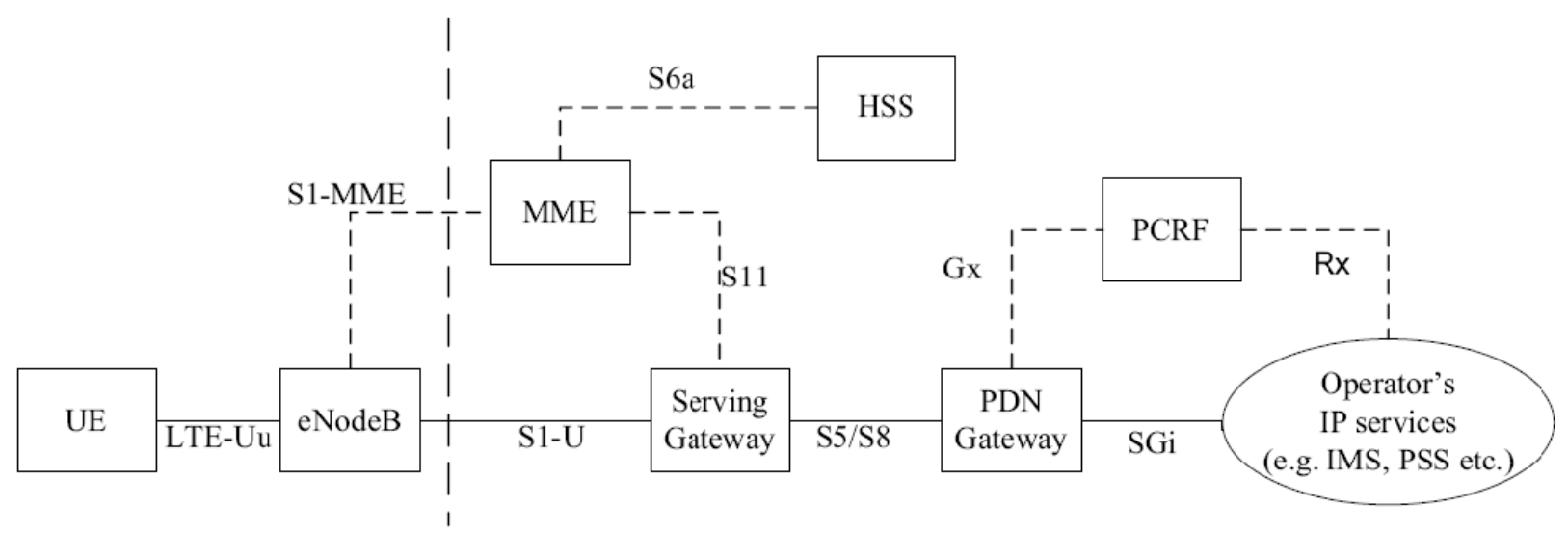

Fig. 1 The LTE network elements 
eNodeB (eNodeBs ${ }^{1}$ ) are normally inter-connected with each other by means of an interface known as X2 (see Fig. 2). The eNodeB also interfaces with the User Equipment (UE). The eNB hosts the PHYsical (PHY), Medium Access Control (MAC), Radio Link Control (RLC), and Packet Data Control Protocol (PDCP) layers that include the functionality of user-plane header-compression and encryption. It also offers Radio Resource Control (RRC) functionality corresponding to the control plane. It performs many functions including radio resource management, admission control, scheduling, enforcement of negotiated UL QoS, cell information broadcast, ciphering/deciphering of user and control plane data, and compression/decompression of DL/UL user plane packet headers [1].

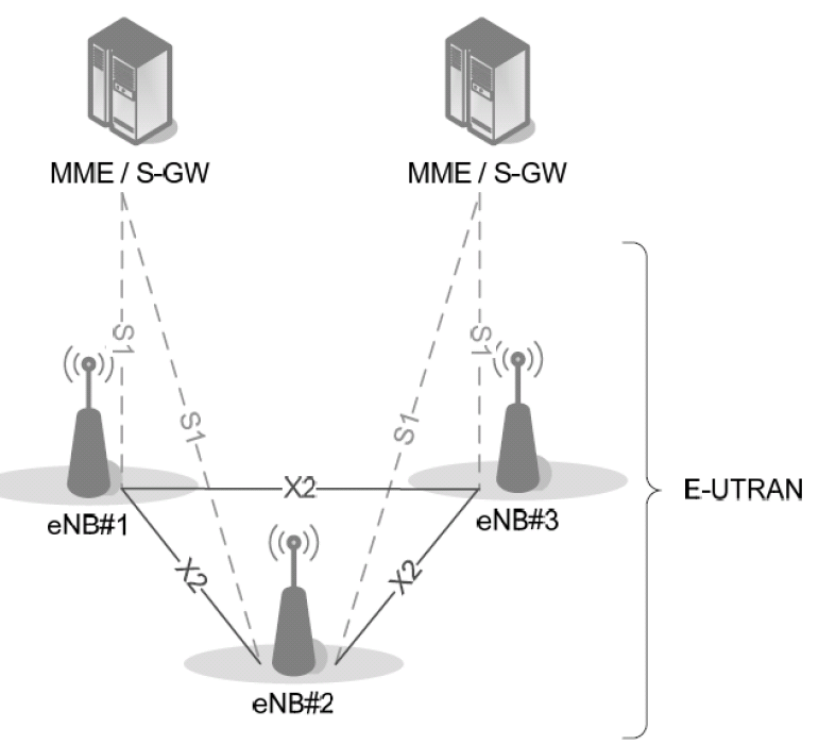

Fig. 2 Overall E-UTRAN architecture

\section{Mobility Management Entity (MME)}

The MME is the key control node for the LTE access network. It is responsible for idle mode UE tracking and paging procedure including retransmissions. It is involved in the bearer activation/deactivation process and it is also responsible for choosing the S-GW (Serving Gateway) for a UE at the initial attach and at time of intra-LTE handover involving Core Network $(\mathrm{CN})$ node relocation. It is responsible for authenticating the user (by interacting with the HSS (Home Subscriber Server)).

\section{Serving Gateway (S - GW)}

The S-GW routes and forwards user data packets, while also acting as the mobility anchor for the user plane during inter-eNB handovers and as the anchor for mobility between LTE and other 3GPP technologies.

\section{Packet Data Network Gateway (P-GW)}

The P-GW provides connectivity to the UE to external packet data networks by being the point of exit and entry of traffic for the UE. An UE may have simultaneous connectivity with more than one P- GW for accessing multiple Packet Data Networks (PDNs). The P-GW performs policy enforcement, packet filtering for each

${ }^{1}$ NodeB is a term used in LTE equivalent to the BTS (Base Transceiver Station) description used in GSM. user, charging support, lawful interception and packet screening. Another key role of the P-GW is to act as the anchor for mobility between 3GPP and non-3GPP technologies such as for instance WiMAX (World Interoprability For Microwave Access) technology.

\section{Home Subscriber Server (HSS)}

The HSS contains users' SAE subscription data such as EPS subscribed QoS profile and any access restrictions for roaming. It also holds information about PDNs to which user can connect.

\section{Policy Control and Charging Function (PCRF)}

The PCRF is responsible for policy control decisionmaking, as well as for controlling the flow based charging functionalities in the Policy Control Enforcement Function (PCEF) which resides in the P-GW.

\section{LTE BEARERS}

In GSM for a standard Circuit Switched (CS) call it is sufficient to assign one UL and DL TCH (Traffic Channel). However in higher technologies (like UMTS and LTE) to meet the throughput requirements one physical channel may not be enough. As the result more than one physical resource can be assigned to one call. This is typical for applications such as VoIP, web browsing, video telephony and video streaming which have special QoS needs. In this case in order to enable differentiation of packet flows based on QoS requirements it is more reasonable to represent the service including the user data transfer from the Layer 2 in the form of a bearer $[1,5]$. The bearer is the concept in 3GPP from UMTS to LTE. The bearers used in LTE are shown in Fig. 3.

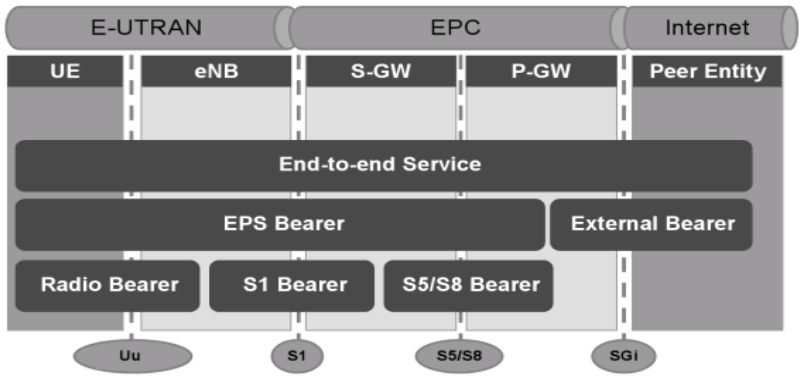

Fig. 3 Bearers used in LTE

As it can be seen there are a few kinds of bearers in LTE. Radio, S1, S5/S8 Bearers and EPS Bearers [1].

Radio bearers carry information on radio interface while S1 bearers exist between eNB and S-GW. S5/S8 bearers are between S-GW and P-GW. The EPS bearer implies radio, $\mathrm{S} 1$ and $\mathrm{S} 5 / \mathrm{S} 8$ bearers while $\mathrm{E}-\mathrm{RAB}$ implies radio and $\mathrm{S} 1$ bearers. There is a one to one mapping between Radio, S1, S5/S8 and EPS bearers.

If a bearer delivers c-plane (signalling) message, that radio bearer will be called Signalling Radio Bearer $(\mathrm{SRB})^{2}$. If it delivers $\mathrm{u}$-plane data, that radio bearer will

${ }^{2}$ There are three kinds of Signaling Radio Bearers in LTE: SRB0 which is for transmitting RRC messages using $\mathrm{CCCH}$ (Common control channels) logical channel. SRB1 which is for transmitting NAS messages over DCCH (dedicated control channels) logical channel. SRB2 which is for high priority RRC messages. Transmitted over DCCH logical channel [1]. 
be named as Data Radio Bearer (DRB) ${ }^{3}$.

How the bearers are mapped into the logical, transport and physical channels can be found in [1].

\section{CALL SET UP IN LTE}

In this chapter call set up in LTE is described in such a way that the focus is only on the parts necessary to understand the philosophy of E-UTRAN data call set up success rate calculation correctly.

The successful call set up consists of two procedures. The simplified description of these procedures is provided in the next text.

First procedure is Random Access (RA) and connection establishment procedure which is used to create a signalling connection between the UE and the EUTRAN. It can be initiated only by the UE sending a RandomAccessPreamble message on the Physical Random Access Channel (PRACH) to the eNB. This message contains a so called contention-free signature used by UE from a set of signatures broadcasted by the system. Consequently a Random Access Response (RAR) message is sent from eNB to UE on a physical DL shared channel (PDSCH). The RAR conveys the identity of the detected preamble, a timing alignment instruction to synchronize subsequent uplink transmission from UE and an initial uplink resource grant for the transmission of the $\mathrm{RRC}$ connection request message. This message in fact represents a signaling connection establishment attempt with a specific reason why the UE is requesting a signalling connection (SRB1) $[1,2]$. Possible reasons are:

- $\quad$ emergency call

- MO signalling

- Mo data

- MT access

- other procedures

Then it comes next signalization between the UE and network in order to activate the signaling radio bearer 1 (SRB1). The successful seizure of physical resources assigned to SRB1 is acknowledged by sending the RRCConnectionSetupComplete message from UE to eNB. This message in addition involves the transfer of the initial uplink non Access Stratum (NAS) message which is consequently sent from eNB to MME/EPC within the S1AP: InitialUE message which triggers the establishment of UE associated S1 logical connection and contains information if initial security and default bearer activation or only dedicated bearer activation is needed (see note 3 ).

Second procedure is initial context set up procedure to establish a radio resource connection and a connection to the core. In UMTS (3G) these two steps carried out one after the other, make for quite a long procedure for location of area updates, call establishments, Packet Data Protocol (PDP) context establishments, etc., leading to a control plane latency which would be unacceptable for LTE. In order to solve the problem a new feature initial context set up has been introduced for LTE. As mentioned

\footnotetext{
${ }^{3}$ Depending whether the bearer is assigned during so called initial context set up or additional set up (see chapter 4) we distinguish between default and dedicated bearers. Based on the nature of QoS, bearers can be classified as Minimum Guaranted Bite Rate (GBR) bearers which can be used for applications as VoIP and non - GBR bearers which do not guarantee any particular bit rate. Default bearers
} are allways non - GBR ones [1].

above the key feature of the initial context set up procedure is that radio access network signaling and core network signaling are in parallel.

The EPC is initiator of this procedure ${ }^{4}$. The EPC sends an S1AP: InitialContextSetupRequest message to eNB which repeats it in a form of RRCConnectionReconfiguration message sent to UE containing a radio resource configuration used to establish a radio/EPS bearer. After sending the message the following procedures are done in parallel:

- Further coordination procedure (authentication, ciphering etc.) which are performed on the SRB1 $[1,2]$.

- And establishment of a radio resource connection, i.e if the radio resource configuration assigned to the radio/EPS bearer has successfully been seizured by UE, the UE sends the RRCConnection ReconfigurationComplete message (which confirms that the requested radio bearer has been successfuly assigned) to eNB which repeats it in a form of S1AP: Initial ContextSetupResponse message (which confirms that the requested EPS bearer has been successfuly assigned) to MME [1,2]. It should be noted that during this phase so called default and non-GBR bearers can only be assigned (see note 2 and 3 in the chapter 3 ). The message exchange related to this point is also performed on SRB1.

Fig. 4 summarizes the above described procedures.

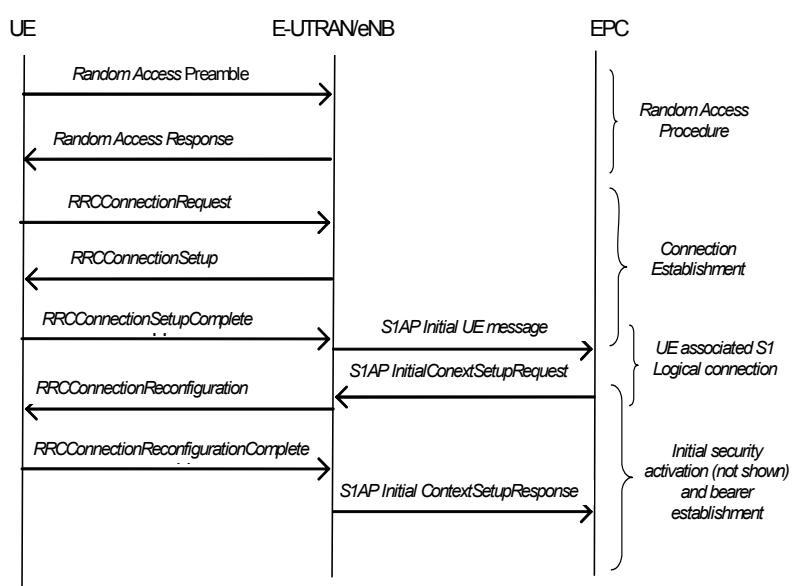

Fig. 4 Message flows for the initial call set up in LTE

After the default EPS bearer has been successfuly assigned there maybe a request from UE for some other, e.g. VoIP service when GBR bearer is needed. This is called as additional EPS bearer activation. For this kind of bearer activation the above message flow can be reused with the exception that random access procedure and initial security activation do not apply as the UE is already in active state. Therefore instead of the S1AP: InitialContextSetupRequest and SIAP. InitialContextSetupResponse between $\mathrm{eNB}$ and EPC the S1AP: E-RABSetupRequest and SIAP: ERABSetupResponse are sent $[1,2]$.

${ }^{4}$ It is understood from E-UTRAN point of view as for E-UTRAN in fact the
initiator of this procedure is MME. But from overall point of view it is initiated by P-GW [1]. 


\section{DEFINITION OF REAL E-UTRAN DATA CALL} SET UP SUCCESS RATE

As mentioned in the previous paragraph the successful data call set up consists of the following parts:

- Successful random access procedure (the result of that is initial uplink resource grant for the consequent RRC connection establishment)

- Successful RRC connection establishment (the result of that is successful assignment of the SB1 resources and transmission of the NAS message into the EPC)

- Successful default EPS bearer assignment

The rest of the procedures like UE associated S1 logical connection, initial security activation and SRB1 drops during call set up due to EPC shall not be counted.

Therefore the Real E-UTRAN Data CSSR is given by the formula:

$$
\text { CSSR }=\frac{\text { EPSBearerAssignments }}{\text { NumRA PreambleExc }}
$$

where NumRAPreambleExc represents the number of sent random acess preambles from UE to eNB related to data calls with excluded ghosts ${ }^{5}$ and cases which later on lead to SRB1 drops or unsuccessful authentication due to EPC or unsuccessful UE associated S1 logical connection, i.e. NumRAPreambleExc = NumRAPreambleData. The EPSBearerAssignments represents the number of successfuly assigned default EPS bearers.

Practical implementation of the Eq. 1 means problem because up to now it is not possible to distinguish between the requests for the data and signalling call in Mobile Terminated (MT) access. In fact when UE sends RRCConnectionRequest with the cause MT access it may represnt both a data or signalling call.

One of the possibilities how to solve this problem could be using of simplified formula:

$$
\operatorname{CSSR}^{*}=\frac{\text { EPSBearerAssignments }}{\text { NumRA Pr eamble }}
$$

where NumRAPreamble represents the total number of random access preambles. Let us consider that NumRAPreamble $=$ NumRAPreambleData + Residuum, where Residuum represents ghosts, random access preambles related to signalling calls and those cases which later on lead to SRB1 drops or unsuccessful authentication due to EPC or unsuccessful UE associated S1 logical connection.

Then the Eq. 2 can be modified to the following form:

$$
\operatorname{CSSR}^{*}=\frac{\text { EPSBearerAssignments }}{\text { NumRAPreambleData }+ \text { Re siduum }} .
$$

Under condition that Residuum/NumRAPreambleData $\leq 20 \%$ the formula can be modified using binomial series as follows:

\footnotetext{
${ }^{5}$ Ghosts are phantom preambles not foreseen for the observed cell. The random access procedure is then started, but will not be successful, because no UE will reply with a RRCConnectionRequest message.

CSSR $^{*} \approx \frac{\text { EPSBearerAssignments }}{\text { NumRAPreambleData }}\left(1-\frac{\text { Re siduum }}{\text { NumRA PreambleData }}\right)$.

Afterwards the absolute error in measurement of data CSSR using Eq. 2 will be

$$
\begin{aligned}
& \Delta=\text { CSSR }^{*}-\text { CSSR }=\frac{\text { EPSBearerAssignments }}{\text { NumRAPr eambleData }}\left(1-\frac{\text { Re siduum }}{\text { NumRAPr eambleData }}\right)- \\
& -\frac{\text { EPSBearerAssignments }}{\text { NumRAPr eambleData }}=-C S S R \cdot \frac{\text { Re siduum }}{\text { NumRAPr eambleData }}
\end{aligned}
$$

In case that Residuum is equal to zero the Eq. 2 provides exactly the data CSSR but this is not real case because in practice the ratio:

Residuum/NumRAPreambleData is on the range of tenths of percent which can lead to the big systematic error. Therefore the mobile operators break away from using of the Eq. 2. In principle the Eq. 2 can be used for calculation of data CSSR only in regions with the Residuum/NumRAPreambleData $\leq 1 \%$.

It seems that the best approach promises indirect calculation of NumRAPreambleData according to model in Fig. 3. In similar way also a GSM Call Set up Success Ratio is proposed to be calulated (see in [4]).

In Fig. 5, RA Procedure Success rate is given by formula:

$$
\text { RASuccRate }=\frac{\text { NumRAR }}{\text { NumRAPreamble }},
$$

where NumRAPreamble represents the total number of valid RandomAccessPreamble messages sent from UE to eNB, NumRAR represents the number of RandomAccessResponse messages sent from eNB to UE (see Fig. 4). The reason why the RA procedure was unsuccessful from E-UTRAN point of view can be lack of resources, i.e. an initial uplink resource grant for the consequent RRC connection establishment can not be done.

RRC Connection Setup Success Ratio is given by formula:

$$
\text { RRCSetupSuccRatio }=\frac{\text { NumRRCConnectionSetupCom }}{\text { NumRRCConnection Request }},(7)
$$

where NumRRCConnectionSetupCom represents the number of RRCConnectionSetupComplete messages sent from eNB to UE and NumRRCConnectionRequest represents the number of RRCConnectionRequest messages sent from UE to eNB (see Fig. 4 and [3]). The reason why the RRC connection setup procedure was unsuccessful can be for example radio access problems, timer expiration in eNB when waiting for RRCConnectionSetupComplete message, protocol error, etc. [2]

\section{SRB1 Success Ratio is given by formula}

$$
\text { SRB1SuccRatio = 1-SRB1DropRatio, }
$$

where SRB1DropRatio is SRB1 drop rate and provides how big part from the total number of SRB1 dropped during the procedures (authentication, ciphering etc.) 


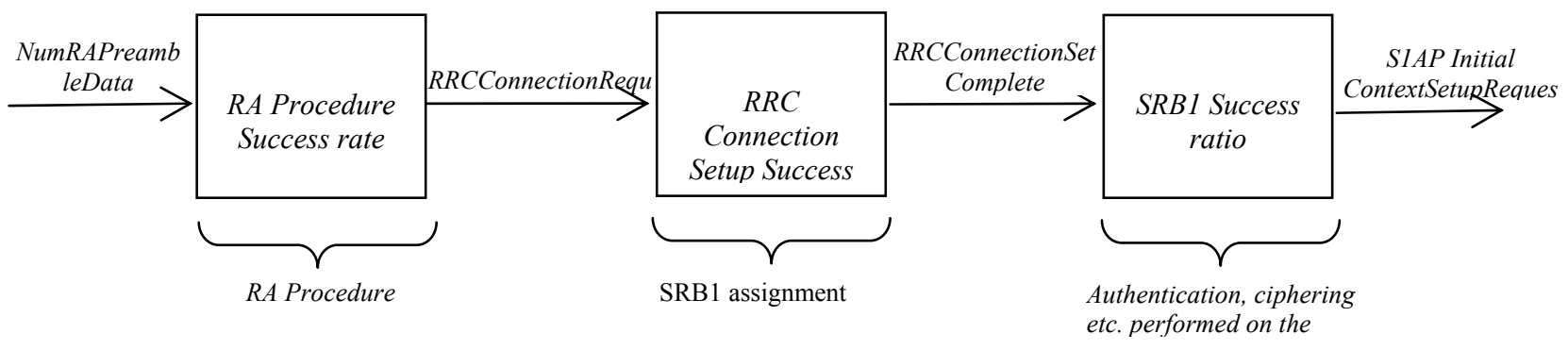

Fig. 5 Model for calculation of NumRAPreambleData

performed on SRB1. It should be noted that only drops due to E-UTRAN are counted within the formula.

From the model in Fig. 5 then we have

S1AP : InitialContextSetup $=$ RASucccRate $\cdot$ RRCSetupSuccRate. -SRB1SuccRate· NumRA Pr eambleData

From Eq. 8 then for NumRAPreambleData we can write that

NumRA PreambleData $=\frac{\text { S1AP : InitialContextSetup }}{\left(\begin{array}{l}\text { RASucccRate } \cdot \text { RRCSetupSuccRate } \cdot \\ \cdot \text { SRB1SuccRate }\end{array}\right)}$

To point out once again with the Eq. 10 the NumRAPreambleData, i.e. the number of sent random acess preambles from UE to eNB related to data calls with excluded ghosts and cases which later on lead to SRB1 drops or unsuccessful authentication due to EPC or unsuccessful UE associated S1 logical connection is estimated. Then including the Eq. 10 into the Eq. 1 it follows that

$\begin{aligned} \text { CSSR }= & \frac{\text { EPSBearerAssignments }}{\text { S1AP }: \text { InitialContextSetup }} \cdot \text { RASucccRate } \cdot \text { RRCSetupSuccRate } . \\ & \cdot \text { SRB } 1 \text { SuccRate }\end{aligned}$

which can be also expressed in the form

$$
\begin{aligned}
\text { CSSR }= & E-\text { RABSetupSuccRateInit } \cdot \text { RASucccRate } \cdot \\
& \text { RRCSetupSuccRate } \cdot \text { SRB1SuccRate }
\end{aligned}
$$

because $\frac{\text { EPSBearerAssignmnets }}{S 1 A P: \text { InitialContextSetup }}$ represents $E$ RABSetupSuccRateInit which is a set up success ratio of initial/default EPS bearers as EPSBearerAssignments is triggered with the S1AP: InitialContextResponse message (see Fig.4 and [3]). Currently the Eq. 12 seems to be best approach for calculation of the E-UTRAN data call set up success ratio. A disadvantage can be higher effort on eNB or equipment (for example an offline tools) where the data CSSR is to be calculated because four KPIs enter the Eq. 12.

With the Eq. 12 the E-UTRAN data call set up success ratio from RA procedure until successful EPS default bearer assignment is calculated. As mentioned in the chapter 4 the default EPS bearer is always classified as non-GBR. However there can be some services like VoIP for which GBR bearer is needed. Those kinds of bearers are activated via additional EPS bearer assignment procedure. Considering a general purpose of call set up success ratio i.e. evaluation of basic procedures relevant from E-UTRAN side until bearer assignment when UE is passing from an idle to an active/connected state the Eq. 12 could seem enough. However there can be some special cases when the only default (non-GBR) bearers are not sufficient from the beginning of UE call (covering all the services assigned to UE). Those can be some special working cases when for example internet browsing (as an eaxample of non GBR service) and VoIP (as an example for GBR service) are requested at the beginning. For such cases it makes sense to evaluate a total E-UTRAN data call set up success ratio. Let us consider the above described procedures from RA until successful default EPS bearer assignment as an event $A$ while additional EPS bearer assignmnet as an event $B$. Then for the Total EUTRAN Data CSSR covering both events we can write

TotalCSSR $=p(A \cap B)$,

where $p$ is a probability operator, i.e. for example $p(A)$ is the probability of the event $A$.

As in our case events $A$ and $B$ are mutual independent we can consider that

TotalCSSR $=p(A) \cdot p(B)$.

Further $p(A)$ is equal to $C S S R$ given by Eq. 12 and

$$
\begin{aligned}
p(B)= & E-R A B \text { SetupSuccRatioAdd }= \\
& =\frac{S 1 A P: E-R A B S e t u p \text { Re sponse }}{S 1 A P: E-R A B S e t u p \text { Re quest }}
\end{aligned}
$$

i.e. $p(B)$ is equal to a set up success ratio of additional EPS bearers, where messages used in the numerator and denominator of the formula are described in the chapter 4 . Hence including the Eq. 14 and 15 into the Eq. 13 for the total data CSSR we receive that

$$
\text { TotalCSSR }=\text { CSSR } \cdot \text { E }- \text { RABSetupSuccRatioAdd }
$$

\section{DISCUSSION AND EXPERIMENTAL RESULTS}

The proposal to calculate the data CSSR only for radio, i.e. E-UTRAN part of the LTE network can be very useful for operators as it is only sensitive to E-UTRAN 
problems. As known currently radio part and core network are optimized and analyzed separately by different Network Planning and Optimization ${ }^{6}$ (NPO) teams. Therefore it is also mandatory to define the KPIs clearly indicating to which part a possible problem is related to. In Fig. 6a an overall LTE CSSR KPI obtained on hourly basis according to Eq. 1 is shown while the E-UTRAN Data CSSR obtained with Eq. 12 is shown in Fig. 6b. It should be noted that the monitored LTE network in this case was not a real one but simulated in a testing laboratory. In Fig. 6a a problem in LTE call set up is visible (averaged CSSR about 95,5\% which is not acceptable) but it is not clear whether the problem is related to radio part or core network part or even both. However with the E-UTRAN Data CSSR as shown in Fig. $6 \mathrm{~b}$ can be visible there is not any problem in radio part as an averaged CSSR is about $99,96 \%$. Concrete in this network a problem related to authentication and ciphering (i.e. EPC one) was introduced in the test which perfectly fits to what shown in Fig. 6 b.

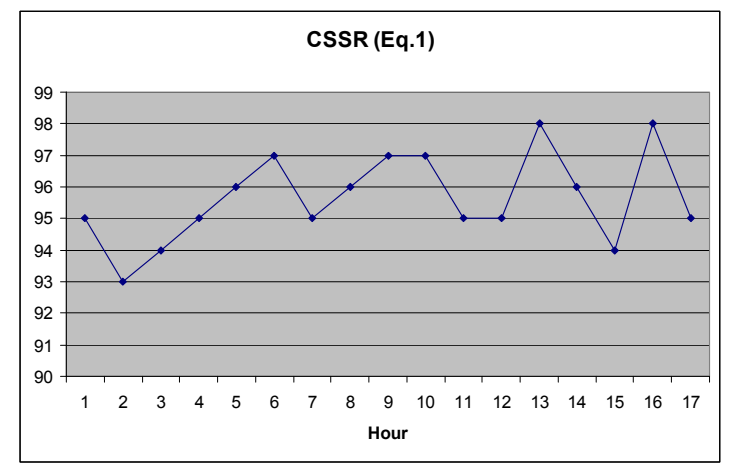

a)

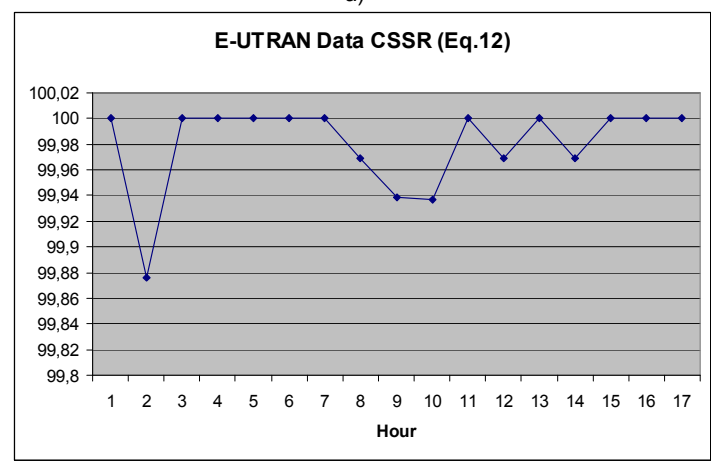

b)

Fig. 6 An CSSR calculated:

a) using the Eq. 1, b) using the Eq. 12

\section{CONCLUSION}

The number of RA preambles for only data calls with excluded ghosts and cases which later on lead to SRB1 drops or unsuccessful authentication due to EPC or unsuccessful UE associated S1 logical connection cannot be measured therefore there is no simple formula for calculation of E-UTRAN Data CSSR. Hence more complex formula into which RA Procedure Success rate, RRC Connection Set up Success Ratio, SRB1 Success Ratio and RAB Set up Success Rate Initial enter must be used. Currently it seems to be the best approach despite higher effort on the processor part in equipment where the E-UTRAN Data CSSR is to be calculated may be expected.

\section{REFERENCES}

[1] SESIA, S. - TOUFIK, I. - BAKER, M.: LTE - The UMTS Long Term Evolution, John Wiley \& Sons, Ltd, 2009.

[2] 3GPP specification: TS 36.413

[3] 3GPP specification: TS 32.450

[4] KOLLÁR, M.: Evolution of the Real Call Set up Success Ratio, Acta Electrotechnica at Informatica, Vol. 8, No. 3, 2008, pp. 53-56.

[5] PAIS, I. - ALMEIDA, M.: End user behavior and performance feedback for service analysis, Intelligence in Next Generation Networks, 2009, ICIN 2009 Conference, Bordeaux, pp. 1-6, October, 2009.

Received April 19, 2011, accepted August 8, 2011

\section{BIOGRAPHY}

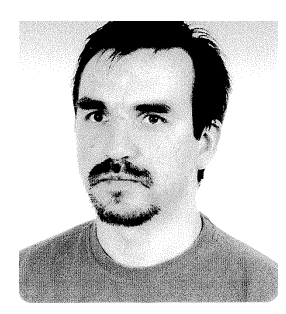

Martin Kollár was born in Spišská Nová Ves, Slovakia, on 7th December 1974. He gained an Ing. (M.Sc.) degree in electronics and multimedia telecommunications from the Faculty of Electrical Engineering and Informatics (FEI), Technical University (TU) of Košice and a Ph.D. degree in measuring techniques from the FEI TU of Košice, in 2000 and 2003, respectively. From 2003 till 2006, he was an assistant professor at the Department of Theory of Electrical Engineering and Measurement FEI TU of Košice. Since 2006 he has been a senior analyst/architect at Siemens Program and System Engineering and working as an external consultant for Nokia Siemens Networks on the O\&M Performance Measurements area for GSM/GPRS/EDGE and LTE. His research interests include performance measurement techniques for GSM/GPRS/EDGE and LTE, nonlinear circuit theory, smart sensors based on a flip-flop circuit and testing the analog-to-digital converters.

\footnotetext{
${ }^{6} \mathrm{NPO}$ ensures that the client objective in terms of service coverage and service quality for mobile networks, broadband networks and broadcast networks are fulfilled on time and on budget.
} 